\title{
Complex Surface Inspection Based on the Minimum Zone Criterion
}

\author{
Tan Gaoshan $^{1,2, a}$, Zhang Liyan ${ }^{1, b}$ \\ ${ }^{1}$ College of Mechanical and Electrical Engineering, Nanjing University of Aeronautics and \\ Astronautics, Nanjing, China \\ ${ }^{2}$ Mathematics \& Physics Engineering Department, Anhui University of Technology, Ma'anshan, \\ China
}

atangaoshan2006@ahut.edu.cn, b'zhangly@nuaa.edu.cn(corresponding author)

\section{Keywords: Surface inspection; Registration; Minimum Zone Criterion}

\begin{abstract}
Although there have been many studies in free-form surface inspection, it remains a difficult problem in application because of different inspection results resulted from various registration methods. Accurate registration is vital in precision manufacturing. Otherwise, rejection of some qualified parts would occur. A new registration method is presented to find the spatial transformation with which the surface error range is minimized. A smooth function is used to approximate uniformly the objective function of the registration to deal with the computational intractability. The limited-memory Quasi-Newton algorithm is prevalent for large-scale optimization problem. It is a differentiable unconstrained optimization method, which only need to provide the function and gradient values of the objective function. The proposed method is easily implemented and works well for dense measurement points. Experiments justify the superiority of the proposed algorithm.
\end{abstract}

\section{Introduction}

It is a necessary step in product manufacturing to determine if a manufactured part meets the design requirements. For the regular parts, the inspection methods and equipments are ready at hand and mature in applications. However, free-form surface is widely used in modern manufacturing. The inspection of these complex parts with multiple freeform surfaces still remains a difficult problem. Numerous researches have been developing in the inspection problem of the free-form surface [1-3]. Firstly, dense points from the part are obtained by various measuring methods. Then, the measured data is aligned with the design model to determine whether the surfaces are out of the tolerance zones. This process is named registration, which figures out a rigid 3D spatial transformation between the design coordinate system (DCS) and the measurement coordinate system (MCS).

No specific standards are available for the complex surfaces registration, so the problem is complex, and different registration methods often lead to different verdict results. The commonly used criterion for geometric model registration is the least squares criterion [4,5]. It minimizes the sum of squared distances from the measured points to its corresponding points on design surfaces. The error distribution is even based on the least square criterion. The mini-max criterion is another criterion for the surface inspection. It seeked the minimization of the maximum error[6]. The sequential quadratic programming (SQP) algorithm was used to solve the optimization model. It is at the cost of doubling the amount of the distance constraints, which result to troubles especially when the number of the measured points is huge. We noticed the minimum zone criterion is intuitively proper for the surface registration problem. It manages to locate the physical contour in the optimum position to minimize the distance between two offset surfaces of the theoretical contour, which embrace all the measurement points. Minimum zone methods for regular surface inspection [7] had been implemented in applications. The modified artificial bee colony algorithm [8] and the ant colony algorithm [7] are used to solve the flatness and cylindrical surfaces registration problem of based on the minimum zone criterion. However, they are very sensitive and they have low computation efficiency in general. The minimum zone criterion conforms to the ISO 
standard [9] for form tolerances, while it is not generally used to complex surface registration for the intractability of its optimization model.

Registration in the complex surface inspection essentially is a large-scale nonlinear optimization problem. To achieve an optimal inspection verdict, our work is to align a complex part with freedom surfaces to its design model based on the minimum zone criterion and find an efficient solution for the optimization problem. The proposed method is mathematically the minimization of the interval distance of the two offset theoretical surfaces. The objective function is discontinuous and non-differentiable, so algorithmically finding the smooth uniformly approximate function of the range function is significant. As an alternative, the aggregation function is derived. As a delectable result, the optimization problem can be efficiently solved using the conventional Quasi-Newton algorithms. The superiority of the proposed method will be demonstrated by the example.

\section{Mathematic Model for the Problem}

Suppose the measured points of the manufactured part be denoted as $P=\left\{P_{i} \mid i=1,2, \cdots, n\right\}$ in MCS, and the design model surface be denoted as $Q$ in DCS. The purpose of the registration is to find the rigid transformation that aligns the points $P_{i}$ with $Q$ in such a way that the distance between two offset surfaces of the theoretical model as small as possible. The two offset models embrace all the measured points. The deviation from the part to the design model can be described by the oriented Euclidean distance from $P_{i}(i=1, \cdots, n)$ to $Q$, which is defined as

$$
d_{i}(R, T)=\left(R P_{i}+T-Q_{i}\right) \cdot n_{i}
$$

where the point $Q_{i}$ is the nearest point on the nominal surface of the measurement point $P_{i}$, which is obtained by the kd-tree search algorithm in this paper; $R \in \square^{3 \times 3}$ is the rotation matrix, $T \in \square^{3}$ is the translation vector, and $n_{i}$ is the normal vector pointing outward of surface $Q$ at the point $Q_{i}$. If Euclidean distance is negative, it represents the point $P_{i}$ is in the design surface, whereas is out the surface. To meet the requirement of the registration proposed in this paper, the optimization model can be naturally formulated as:

$$
\min _{R \in \square^{3 \times 3}, T \in \square^{3}}\left(\max _{i \in I} d_{i}(R, T)-\min _{i \in I} d_{i}(R, T)\right)
$$

where $I=\{1,2, \cdots, n\}, \mathrm{n}$ is the number of the measured points. Rotation matrix $R$ and translation vector $T$ are as follows:

$$
\begin{gathered}
R=\left[\begin{array}{ccc}
\mathrm{c} \theta_{\mathrm{z}} \mathrm{c} \theta_{\mathrm{y}} & \mathrm{c} \theta_{\mathrm{z}} \mathrm{s} \theta_{\mathrm{y}} \mathrm{s} \theta_{\mathrm{x}}-\mathrm{s} \theta_{\mathrm{z}} \mathrm{c} \theta_{\mathrm{x}} & \mathrm{c} \theta_{\mathrm{z}} \mathrm{s} \theta_{\mathrm{y}} \mathrm{c} \theta_{\mathrm{x}}+\mathrm{s} \theta_{\mathrm{z}} \mathrm{s} \theta_{\mathrm{x}} \\
\mathrm{s} \theta_{\mathrm{z}} \mathrm{c} \theta_{\mathrm{y}} & \mathrm{s} \theta_{\mathrm{z}} \mathrm{s} \theta_{\mathrm{y}} \mathrm{s} \theta_{\mathrm{x}}-\mathrm{c} \theta_{\mathrm{z}} \mathrm{c} \theta_{\mathrm{x}} & \mathrm{s} \theta_{\mathrm{z}} \mathrm{s} \theta_{\mathrm{y}} \mathrm{c} \theta_{\mathrm{x}}-\mathrm{c} \theta_{\mathrm{z}} \mathrm{s} \theta_{\mathrm{x}} \\
-\mathrm{s} \theta_{\mathrm{y}} & \mathrm{c} \theta_{\mathrm{y}} \mathrm{s} \theta_{\mathrm{x}} & \mathrm{c} \theta_{\mathrm{y}} \mathrm{c} \theta_{\mathrm{x}}
\end{array}\right] \\
T=\left(t_{\mathrm{x}}, t_{\mathrm{y}}, t_{\mathrm{z}}\right)^{\mathrm{T}}
\end{gathered}
$$

where $\mathrm{c}$ and $\mathrm{s}$ represent cosine and sine function respectively, $\theta_{\mathrm{x}}, \theta_{\mathrm{y}}, \theta_{\mathrm{z}}$ are the rotation variables. The optimization model is established on the minimum zone criterion, which conforms to the ISO standard.

Let $X=\left[\theta_{x}, \theta_{y}, \theta_{z}, t_{x}, t_{y}, t_{z}\right]^{\mathrm{T}}$ be the transformation variables, then Eq. (1) can be written as a function of the variable vector $X$,

$$
d_{i}(X)=\left(R(X) P_{i}+T(X)-Q_{i}\right) \cdot n_{i}
$$

Hence, the problem (2) can be briefly written as

$$
\min _{X \in \square^{6}}\left(\max _{i \in I} d_{i}(X)-\min _{i \in I} d_{i}(X)\right)
$$

\section{The Aggregation Function Approximation and Its Optimization}

The objective function in (6) is the range function. It is non-differential. Generally, this kind of problem can be solved by some direct/heuristic search algorithms. Their computation efficiency and accuracy are unsatisfactory relative to the traditional differentiation optimization algorithms. 
Moreover, the heuristic algorithms can not ensure numerical convergence.

The aggregation function can uniformly approximate the max function by an explicit twice-differential function as the control parameter introduced tends to infinity. We get the aggregate function approximation of the range function

$$
F_{p}(X)=\frac{1}{p} \cdot \ln \left[\sum_{i=1}^{n} e^{p \cdot d_{i}(X)}\right]+\frac{1}{p} \cdot \ln \left[\sum_{i=1}^{n} e^{p \cdot\left(-d_{i}(X)\right)}\right]
$$

Where $p$ is a positive control parameter, which tends towards infinity in theory. Thus the optimization model is converted to the following minimization problem

$$
\min _{X \in \square^{6}} F_{p}(X)
$$

The Broyden-Fletcher-Goldfarb-Shanno (BFGS) algorithm is efficient and prevalent for this type of unconstrained nonlinear programming problem. As a representative Quasi-Newton algorithm, the BFGS algorithm cares only about calculation of the value and the gradient of the objective function. In order to adapt to the big data in precision inspection, limited-memory Broyden-Fletcher-Goldfarb-Shanno (L-BFGS) algorithm is used, which is a low-memory extension of the BFGS algorithm. Therefore, the optimal registration is implemented by integrating the L-BFGS subprograms into the $\mathrm{VC}++$ environment. The following figure shows the algorithm flow diagram. Fig. 1 is the workflow of the proposed minimum zone registration method.

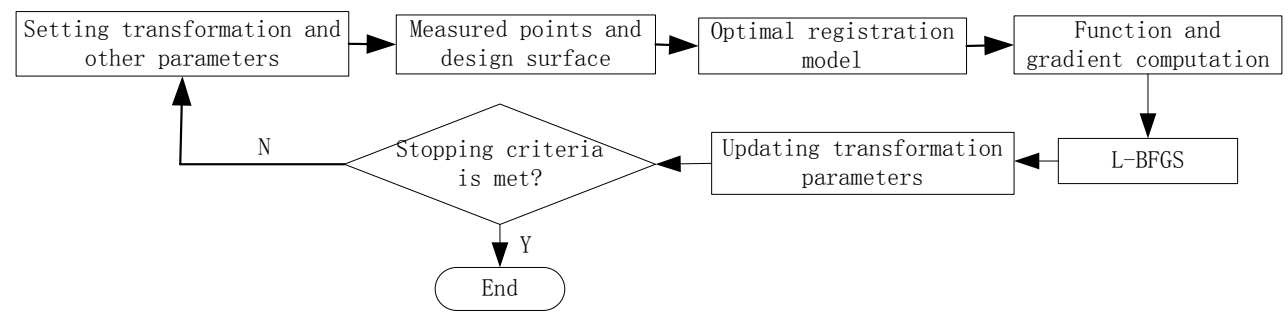

Fig.1. Workflow of optimal registration process

\section{Experiment Results on a Sheet Metal Part}

This experiment concerns the error distribution of a real sheet metal part. The part was made with the stamping process. Since the part was in complex freeform surfaces and the error distribution was hard to predict because of the inevitable deformation, it was critical to algorithmically align the measured point with the nominal model ahead of the assembling process. We first got the initial relative pose by using the "best fit" function of the popular commercial software Imageware ${ }^{\circledR}$. Then, the registration is realized by the least square method and the minimum zone method proposed in this paper. Fig. 2 is the result of the minimum zone method. The range error is $1.744 \mathrm{~mm}$, which is nearly $0.1 \mathrm{~mm}$ less than the value in the least square method. Table 1 lists the error statistic obtained by the tested methods. The magnitudes of the maximum errors in our method are smaller whatever positive or negative. Positive standard errors show less volatility in our method than in the least square, since the standard deviations are $0.206 \mathrm{~mm}$ and $0.233 \mathrm{~mm}$ respectively. Meanwhile, the discreteness of the negative error is nearly equivalent in the two methods. Of course, the total standard deviation is bigger in the minimum zone method than in the least square method.

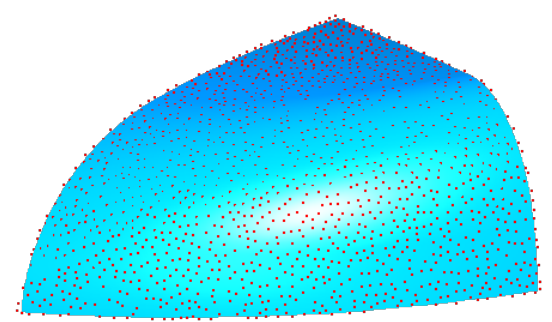

Fig.2. Registration of the real sheet metal part 
Table 1. Comparisons of the minimum zone registration and the least square registration on the sheet metal parts

\begin{tabular}{lcc}
\hline \multicolumn{1}{c}{ Errorlmethods } & Least square method & $\begin{array}{c}\text { Minimum zone } \\
\text { method }\end{array}$ \\
\hline Range error & 1.8331 & 1.7437 \\
Minimum error & -0.5043 & -0.4909 \\
Maximum error & 1.3288 & 1.2528 \\
Total standard error & 0.1992 & 0.2283 \\
Negative Standard error & 0.1278 & 0.1289 \\
Positive Standard error & 0.2333 & 0.2057 \\
\hline
\end{tabular}

\section{Conclusion}

A new machining quality evaluation and registration technique is proposed for complex parts. Taking the smallest space between the parts and the design model as the first concern, we formulated the surface registration as an unconstrained non-differentiable minimization of the range function. Then, the smooth uniformly approximate aggregation function is used to substitute the range function. The problem was efficiently and stably solved with the conventional Quasi-Newton algorithms. The tested result indicated that the proposed method tends to output preferable inspection result and helps reduce false rejections in the geometry quality inspection in practice.

\section{Acknowledgement}

The research was sponsored byJiangsu Innovation Program for Graduate Education (KYLX-0309, the Fundamental Research Funds for Central Universties) and the Specialist Foundation in Civil Aircraft (MJ-G- 2011 -24).

\section{References}

[1] $\mathrm{Li} \mathrm{YD}$ and $\mathrm{Gu} \mathrm{PH}$. Free-form surface inspection techniques state of the art review[J]. Computer-Aided Design,2004, 36:1395-1417.

[2] Savio E, Chiffre LD, Schmitt R. Metrology of freeform shaped parts[J]. Annals of the CIRP,2007, 56/2: 810-835.

[3] Paolo Minetola. The importance of a correct alignment in contactless inspection of additive manufactured parts[J]. International Journal of Precision Engineering and Manufacturing,2012, 13(2):211-218.

[4] Besl PJ, McKay ND. A method for registration of 3-d shapes[J]. IEEE Transactions on PAMI, 1992, 14(2): 239-256.

[5] Wen XL, Zhao YB, Wang DX, Zhu XC, Xue XQ. Accurate evaluation of free-form surface profile error based on Quasi Particle Swarm Optimization algorithm and surface subdivision[J]. Chinese Journal of Mechanical Engineering, 2013, 26(2): 406-413.

[6] Sun YW,Wang XM, Guo DM, Liu J. Machining localization and quality evaluation of parts with sculptured surfaces using SQP method[J]. International Journal of Advanced Manufacturing Technology, 2009, 42: 1131-1139.

[7] Liu J, Wang GL, Pan XD. Minimum-zone form tolerance evaluation for cylindrical surfaces using adaptive ant colony optimization[J]. Journal of Computational Information Systems, 2011, 7(12):4480-4490.

[8] Luo J, Wang Q, Fu L. Application on modified artificial bee colony algorithm to flatness error evaluation[J]. Optics and Precision Engineering, 2012, 20(2):422-430.

[9] International Standard Organization. ISO/TS 17450-1-2007, Geometrical product specifications (GPS)- General concepts- Part 1: Model for geometrical specification and verification[S]. Switzerland: ISO copyright office, 2007. 